\title{
The History of Innovation in Radio Astronomy
}

\author{
R D Ekers ${ }^{1}$
}

CSIRO Astronomy \& Space Science

Vimiera \& Pembroke Roads, Marsfield, NSW 2121

Australia

E-mail: Ron.Ekers@csiro.au

\begin{abstract}
This meeting includes many stories and scientific results from the radio astronomy community who have gathered here in Kerastari. To provide context for these presentations I have included this brief summary of the history of innovation in radio astronomy.

I start with the serendipitous beginnings of radio astronomy and the development of the radio telescope dishes. Early scientific discoveries included the identification of radio stars as extragalactic radio sources and this led to the discovery of quasars whose enormous luminosity required accretion of matter onto black holes in the centre of galaxies to provide the source of energy.

The need for high angular resolution at the long radio wavelengths resulted in the development of aperture synthesis radio telescopes using techniques analogous to the X-ray tomographic imaging used to image the internal structure of the Antikythera Mechanism. The ultimate radio resolution uses the VLBI techniques which in turn provide the highest precision data for modern astrometry.

We can see how this new technology driven science was slowly adopted by the scientific community and we can look at the environment which enabled such rapid development with no less than 6 Nobel prize discoveries in this one narrow field of astronomy. Finally, I include some contributions of Derek De Solla Price whose analysis of the process of discovery and the evolution of technology applies very well to the development of radio astronomy.
\end{abstract}

From Antikythera to the Square Kilometre Array: Lessons from the Ancients, Kerastari, Greece

12-15 June 2012

\footnotetext{
$1 \quad$ Speaker
} 


\section{Introduction}

This meeting includes many stories and scientific results from the radio astronomy community who have gathered here in Kerastari for this meeting. To provide context for these presentations I have included this brief summary of the history of innovation in radio astronomy. In 1609 Galileo built a telescope and was the first person to see the moons of Jupiter. It was more than 300 years before mankind saw the universe through another wavelength window. The field of radio astronomy began in the 1930s so it is still less than a century old and this has made it possible to document the development of this new science almost entirely from the direct accounts of the pioneers.

\section{Beginning of radio astronomy}

In the course of trying to identify the source of interference to trans-Atlantic telephone communications Karl Jansky, working at the Bell Telephone Laboratory, discovered cosmic radio emission in 1933 [1]. An unexpected source of noise (Jansky's Cosmic Hiss) was peaking each day but the peak signal arrived 4 min earlier each day and Jansky realised that this corresponded to sidereal (star) time and hence it must have extraterrestrial origins. The reaction from Bell Labs was underwhelming: "so faint not even interesting as a source of radio interference!" Once he had determined that the interference was "of extraterrestrial origin" there was little support from the Bell Telephone Laboratory to further pin down the location in space. Sullivan [2] has noted that while a few astronomers expressed interest at the time, for the majority the world of decibels and superheterodyne receivers was too far removed from their world and Jansky had died before the importance of his discovery was appreciated. This discovery, enabled by technology, was completely unexpected.

Although the scientists ignored Jansky's discovery, in December 1938 science fiction writer Edmond Hamilton wrote "The Cosmic Hiss" based on Jansky’s observation of cosmic hiss [3].

\section{The Dishes}

In 1937 one successful amateur radio ham, Grote Reber, undertook the challenge to understand what Jansky had found. He built a home-made \$2000 32 foot parabolic dish in his mother's backyard in Wheaton, Illinois, and started looking for this Jansky emission. At first he looked at shorter wavelengths than used by Jansky because the only type of natural radio emission known was thermal and thermal radio emission would be stronger at shorter wavelengths. But nothing was seen at the shorter wavelengths and Reber changed to longer and longer wavelengths until he finally detected cosmic static similar to that seen by Jansky [4]. This radio emission had to be of non-thermal origin but there was no concept of non-thermal astronomical emission at any wavelength so things were even more puzzling. It was not until 13 
years later in 1950 that the synchrotron radiation from high energy particles in space (cosmic rays) was understood and these results could be integrated into the larger scientific world view and the strange world of extra terrestrial radio noise became part of astronomy [5].

The 32-foot parabolic antenna built in Wheaton, Illinois, in 1937 was the forerunner of the parabolic dishes which have played a dominant role in the development of radio astronomy and was the forerunner of the now ubiquitous parabolic dishes seen on many house roofs and radio communication complexes. In the 1950s at Jodrell Bank, U.K., Bernard Lovell started work on the 218-foot dish. A decade later the CSIRO Radiophysics group in Australia built a 210-foot dish now known as the Parkes radio telescope. In 1963 at Arecibo, Puerto Rico, the US constructed the largest single aperture reflecting dish ever built. This has a 1000 foot diameter but is a fixed spherical reflector with correcting optics and a moveable focus. The Max Planck Institute, 100-metre dish in Effelsberg near Bonn was next. It is a classical steerable parabolic dish completed in 1973. The 100-metre Green Bank Telescope (GBT), replacing an older transit dish, is the last of the giant moveable dishes to be constructed. It commenced operation in 2000. Unlike its predecessors, the GBT is an off-axis segment of a parabola. At the same Greenbank observatory you will now also find the original Grote Reber dish which was reassembled there by Reber in 1960 .

The Chinese FAST radio telescope now under construction was originally conceived as an element of a future Square Kilometre Array (SKA), see section 9. It is a single very large, 500$\mathrm{m}$, Arecibo-like dish. It is being built in the karst region of Guizhou province in China and uses an innovative stretched membrane structure to locally deform the spherical dish into a parabolic shape rather than using a line feed or a large correcting secondary mirror as is done at Arecibo. It is expected to be completed by 2016.

\section{The story of the radio stars, quasars, and black holes}

\subsection{Cygnus A, strongest radio source in the sky}

In 1946 Stanley Hey, one of the co-discoverers of radio emission from the sun in the UK during WWII, found that one of the strong sources of radio emission was fluctuating on time scales of 10-30 seconds [6]. He correctly concluded that the source must be small diameter, less than a light minute in size. This was the size of a star but there was no optical counterpart so what was it? Was the whole plane of the Galaxy made of such radio stars?

\subsection{The Cliff interferometer and the discovery of the radio galaxies}

In 1946 at Dover Heights near Sydney a telescope was constructed on the cliff to measure the interference between the direct waves and those reflected by the sea (a Lloyd's mirror). This cliff interferometer was built to locate the origin of the solar radio emission and to identify the radio stars. The idea of a cliff interferometer came from multiple path interference already seen in ship-borne radar in WWII and used to improve positional information. John Bolton and his colleagues [7] at CSIRO in Australia were able to measure positions accurately enough to identify three of the strongest of the mysterious discrete sources of radio emission which up until this time were thought to be radio stars. One was the Crab nebula, the remnant of a star 
that the Chinese had seen explode 900 years ago. The other two were an even greater surprise. Centaurus A and Virgo A (strongest sources of radio emission in the constellations of Centaurus and Virgo) had conspicuous bright optical identifications which were galaxies - not stars! Galaxies, far outside our own milky way but undergoing such a violent explosion that they were among the brightest objects in the radio sky and became the most luminous sources known in the universe. This discovery, with some help from the now very enthusiastic optical astronomers at Mt Palomar in the US, led to the eventual identification of the strongest of all the radio sources, Cygnus A. It was found to be a very faint galaxy so distant that it was immediately obvious that these new radio telescopes were probing the most distant reaches of the universe!

Prior to 1963 the extragalactic radio sources were almost all identified with giant elliptical galaxies. This was all to be changed in an unexpected way. Astronomers following essentially the same procedure used for eclipse prediction with the Antikythera Mechanism calculated when the radio source 3C273 would be occulted by the moon. The occultation occurred as predicted and was observed by Cyril Hazard [8] using CSIRO's Parkes radio telescope. It showed an unresolved core with a 20" spike. The morphology and position clearly identified this strong but previously unidentified radio source with a bright 13 magnitude star and a wisp (jet) of optical emission in the same location as the radio spike. Martin Schmidt obtained an optical spectrum of the star and interpreted the lines as having a redshift corresponding to a recession velocity of 0.15 of the speed of light [9] , implying unprecedented optical luminosity comparable to an entire galaxy but coming from a region as small as a star. This was the first quasar.

This discovery was the trigger for the first Texas Symposium on Gravitational Collapse and Relativistic Astrophysics [10]. Only a black hole could provide the energy required from such a small volume. This was a paradigm shift in astronomy and the process of unravelling the role of black holes in the evolution of the Universe continues to this day.

\section{History of Fourier synthesis imaging in radio and $x$-ray}

Michelson had already used an optical interferometer to measure the size of stars in 1891 [11]. He defined fringe visibility and gave the Fourier equations but didn't call it a Fourier transform. In medical x-ray imaging stereo x-ray pairs were used to estimate 3-D structures and between 1930 and 1938 3-D X-ray tomography was developed using analogue devices to do back projection summation. In 1930 the van Cittert-Zernike theorem was published and is now considered the basis of Fourier synthesis imaging although it played no role in the early radio astronomy developments.

X-ray diffraction in crystals was discovered in 1912. By 1936 Fourier synthesis calculations had become routine in X-ray crystallography using Lipson \& Beevers strips to make the calculations by hand. In 1939 Bragg's X-ray crystallography group was flourishing at the Cavendish Laboratory and would later catalyse the Cambridge radio astronomy group's imaging developments. 


\section{Aperture synthesis radio telescopes}

Because of the long radio wavelengths it was realised that interferometers with large separation between the elements would be required to obtain high enough angular resolution to determine the origin of the radio waves. Two of the main pioneering groups were at the University of Cambridge in the U.K., led by Martin Ryle, and at the CSIRO (then CSIR) Division of Radiophysics in Sydney, Australia, led by Joe Pawsey. Both groups used the WWII radar technology to build astronomical instruments.

\subsubsection{Australian group}

In 1947 Joe Pawsey, who had done his PhD with Jack Ratcliffe at Cambridge, joined the CSIRO Radiophysics Laboratory in Sydney and with his colleagues used sea interferometer fringes to co-locate solar emission with sunspots [12]. The first published suggestion that it would be possible to synthesise an image of the radio sky by measuring a range of Fourier components was made by Lindsay McCready, Joe Pawsey and Ruby Payne-Scott [13]. However, this technique was impractical with the cliff interferometers they were using, and was not suitable for imaging solar bursts which were strongly variable in both time and frequency. The first observations using a range of Fourier components measured with an interferometer with moveable elements were made at the Cavendish Laboratory.

In Australia the main focus was on solar imaging. The sun is a strong source but has a complex and time variable structure requiring good instantaneous measurements of the Fourier components. For this reason the Australian arrays followed an evolutionary path with large numbers of relatively small elements. In 1951 Chris Christiansen built the Potts Hill grating array with 32 6-foot diameter dishes near Sydney, Australia. By 1955 the first earth rotation synthesis image was obtained by Christiansen and Warburton [14]. In 1967 Paul Wild built a 3 $\mathrm{km}$ diameter circle of 96 3-metre dishes which made moving images of the radio sun. It operated for 17 years from 1967 and resolved many of the questions about the nature of solar bursts [15].

\subsubsection{Cambridge group}

In 1945 Martin Ryle joined Cavendish laboratory and used WWII radar technology for radio astronomy. He introduced the use of a Michelson interferometer to measure the angular diameter of the source of the radio emission [16] and invented what is now called a correlation (multiplying) interferometer [17].

After early experiments observing the sun Ryle's group in Cambridge moved their focus to the observation of radio stars. These sources were static but much weaker than the sun so the arrays evolved along a different path. They used moveable antennas and earth rotation to build up the Fourier components over time and they needed much larger elements to achieve the sensitivity required for the fainter sources. This evolution culminated in the construction of the One-Mile Telescope in 1963 [18]. 


\subsubsection{Earth rotation synthesis}

In June 1961 radio astronomers at the Cavendish Laboratory in Cambridge, UK, used 4C aerials operating at $178 \mathrm{MHz}$ to make a radio source survey of the North Pole region using the earth's rotation to fully sample the aperture plane [19]. Computations and graphical display used EDSAC 2 which was the first use of a digital computer for radio astronomy imaging. The $\mathrm{X}$-ray crystallographers in the Cavendish laboratory had developed the necessary Fourier transform programmes. This was seven years after Christiansen \& Warburton first demonstrated the earth rotation synthesis with an observation of the quiet sun using an array of small dishes in Australia [14]. However, the Australian group took many months to calculate by hand the Fourier transforms for one image and the method was considered impractical at the time.

\subsubsection{Calculating Fourier Transforms}

The means and speed for calculating Fourier transforms has had a major impact on the development of indirect imaging. Since 1936 Fourier transform had been done by hand calculations using Lipson-Beevers strips. A Fourier transform of a 25x25 array to 2 digits could be calculated by 1 person in about 24 hours. In 1949 Maurice Wilkes used EDSAC 1 to do a 38 point transform Fourier transform in 15 hours, demonstrating that it was possible in principle to use an electronic computer but it was impractical for realistic problems. In 1953 a punched card tabulator could be used to Fourier transform a $25 \times 25$ array to 3 digits in 14 hours. However, this took 4 operators [20]! Finally, in 1958 EDSAC 2 was successfully used by the radio astronomers at Cambridge to calculate the Fourier transforms for radio images of the North Pole [19]. In 1984 the Fourier transform was implemented on a single MMIC chip, enabling technology for the 802.11 wireless internet.

Timing tests in 2012 for a modern computer (Ben Humphries, private communication) doing a 32 bit floating point 25x25 Fast Fourier Transform (FFT) were $1.3 \times 10^{5}$ FFTs per second for a 83 GFLOPS laptop and an estimated $1.6 \times 10^{11}$ FFTs per second for the fastest supercomputer.

It is interesting to see where the Antikythera Mechanism, sometimes referred to as the first computer, fits into this sequence. John Reynolds (see talk in this meeting) estimates the calculations required for the Antikythera Mechanism to compute the lunar and planetary positions with its pin slot arrangement to approximate an epicycloid would require 55 floating point operations (FLOPs). Assuming the operator rotated the handle once per second this translates to a 55 floating point operations per second (FLOPS) computer. Wikipedia notes that "a simple calculator needs about 10 FLOPS to be considered functional" (John Reynolds, private communication). 


\section{Aperture Synthesis Telescopes}

\subsection{Cambridge - 1960}

In 1962 the Cambridge group went on to build the One-Mile Telescope [18], and the 5km Telescope in 1971. Martin Ryle was awarded the Nobel Prize in 1974 "For his observations and inventions, in particular for the aperture synthesis technique".

\subsection{Westerbork - 1970}

The forerunner of the Westerbork telescope was the Benelux Cross, a joint NetherlandsBelgium project initiated by Professor Jan Oort to use the radio astronomy source counts for cosmology. By 1964 the Benelux Cross project had become a purely Dutch affair. The design was drastically modified under the influence of Jan Hogbom, a recent $\mathrm{PhD}$ graduate from Ryle's group in Cambridge, and Chris Christiansen, from CSIRO in Sydney. The Benelux Cross was transformed into the Westerbork Synthesis Radio Telescope (WSRT) which combined aspects of aperture synthesis using moveable elements and earth rotation synthesis, from Cambridge, with the grating array concepts, from Australia [21].

The equally spaced telescopes in the Westerbork Synthesis Radio Telescope (WSRT) generated a number of identical interferometer pairs (redundant spacings) and these led to the development of an atmospheric calibration procedure related to the methods of adaptive optics [22]. In 1996 John O'Sullivan, who had moved to CSIRO in Australia, combined this concept with the implementation of a digital Fourier Transform on a single MMIC chip to build the first wideband wireless internet system now known as the 802.11 standard. During this Kerastari meeting John and his colleague David Skellern discuss how basic research in radio astronomy led to this innovative new technology.

\subsection{VLA New Mexico - 1980}

In 1965 a proposal to construct the VLA with 27 25-metre dishes was submitted to the US National Science Foundation (NSF). The VLA construction commenced in 1972 and it was formally inaugurated in 1980 [23]. The VLA has been the most productive ground based telescope ever built at any wavelength in both its number of publications and number of citations. After 30 years with only minimal upgrades the VLA has now had a major upgrade and the Expanded VLA (EVLA) is now coming into operation with 5 to 20 times greater sensitivity, almost complete frequency coverage, and greatly enhanced spectral capability [24]. This illustrates the impact of improved technology even though the collecting area has not changed.

\subsection{VLBI}

In 1967 a new technique of interferometry was developed in which the telescopes were separated by such a large distance that it is necessary to operate them independently with no real-time connection. Instead they used magnetic tape recorders and atomic clocks. The technique is called very-long-baseline- interferometry (VLBI) and is now used to make the 
highest resolution images and the most precise astrometric measurements in astronomy. In many ways this is the modern and very surprising successor of the Antikythera Mechanism.

\section{Derek J de Solla Price}

Derek De Solla Price worked as a teacher of applied mathematics at Raffles College (University of Singapore) in 1948. It was there that he formulated his theory on the exponential growth of science. The idea occurred to him when he noticed the growth in stacks of the Philosophical Transactions of the Royal Society between 1665 and 1850. It was also during the same period that he was studying the Antikythera mechanism, intrigued by the reasons why this advanced technology had disappeared [25].

\subsection{Technology leads scientific discoveries}

In 1960 de Solla Price [26] applied quantitative measurement to the progress of science (scientometrics) and reached the conclusion that most scientific advances follow laboratory experiments. His analysis also showed that the normal mode of growth of science is exponential. In 1981 Martin Harwit made a similar analysis of discoveries in astronomy and again showed that the most important were the result of technical innovation [27]. Martin noted that the discoveries peak soon after new technology appears, usually within 5 years of the technical capability.

A rather simplified conclusion to draw from this is that any field which has not maintained an exponential growth has now died out, so current active research areas are all still in an exponential growth phase. Furthermore, to maintain the exponential the continual introduction of new technology is required since just refining existing technology plateaus out.

\subsection{Exponential growth in science}

A famous example which illustrates this exponential growth very well is the rate of increase of operating energy in particle accelerators by Livingston and Blewett [28]. Starting in 1930, each particle accelerator technology provided exponential growth up to a ceiling when a new technology was introduced. The envelope of the set of curves is itself an exponential with an increase in energy of $10^{10}$ in 60 years. This has been recently updated by Riesselmann to include the Large Hadron Collider [29]. This example of exponential growth, originally presented by Enrico Fermi in 1954, has become known as the 'Livingstone Curve'.

To this we can add the now famous 'Moore's Law' for computing devices (more precisely for transistors on a chip). In 1965 Gordon Moore (co-founder of Intel) noted that the transistor density of semiconductor chips doubled roughly every 1-2 years [30]. This was later refined to doubling every 18 months and this exponential growth has been maintained for the last 40 years [31].

\subsection{Radio Telescope Sensitivity}

Figure 1 plots the sensitivity of telescopes used for radio astronomy since the discovery of extra-terrestrial radio emission in 1940. It has been exponential with an increase in sensitivity 
of $10^{5}$ since 1940 , doubling every three years. Also in this case we can see particular radio telescope technologies reaching ceilings and new technologies being introduced e.g., the transition from huge single dishes to arrays of smaller dishes in the 1980s.

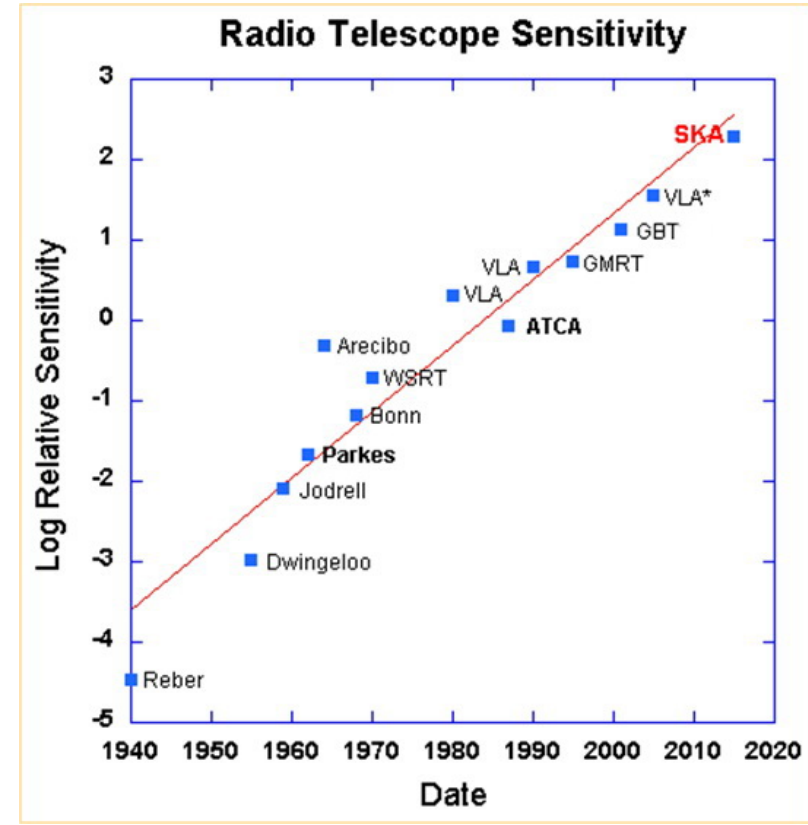

Figure 1: Radio Telescope Sensitivityfor continuum point sources vs. time. Points are the relative continuum point source sensitivity when the telescopes were built, or after major upgrades.

VLA* is the JVLA upgrade. SKA is the proposed sensitivity for a telescope which has not yet been built (see Section 9)

\section{The Square Kilometre Array (SKA) Project}

De Solla Price argued that if the improvement in sensitivity has reached a ceiling the rates of new discoveries will decline and the field will become uninteresting and die out. On the other hand, if we can shift to new technology or find new ways to organize our resources the exponential increase in sensitivity can continue. Do we have such new technology to continue the exponential improvement? In radioastronomy the combination of transistor amplifiers and their large scale integration into complex systems which can be duplicated inexpensively provides one of the keys for change. The other key technology is the computing capacity to apply digital processing at high bandwidth thereby realizing processes such as multiple adaptive beam formation and active interference rejection in ways not previously conceivable. Finally, the move to international facilities such as the proposed SKA will also be needed to avoid the resource ceiling [32].

Did the development of devices like the Antikythera Mechanism die out because the technology at the time had reached the ceiling for mechanical complexity? 


\section{References}

[1] K. Jansky, Electrical disturbances apparently of extraterrestrial origin, Proc. IRE 2,1 387, 1933.

[2] W.T. Sullivan, Cosmic Noise, Cambridge University Press, U.K., 2009.

[3] E. Hamilton, The Cosmic Hiss, in Thrilling Wonder Stories, 90-97, Standard Magazines,1938.

[4] G. Reber, Cosmic static, Proc. IRE 28, 68-70, 1940.

[5] H. Alvén H, N. Herlofson, Cosmic radiation and radio stars Phys. Rev. 78, 616, 1950.

[6] J.S. Hey, S.J. Parsons, J.W. Phillips, Fluctuations in cosmic radiation at radio frequencies, Nature 158, 234, 1946

[7] J.G. Bolton, G.J. Stanley G J, O.B. Slee O B, Positions of three discrete sources of galactic radio-frequency radiation, Nature 164, 101, 1949.

[8] C. Hazard, M.B. Mackey, A.J. Shimmins, Investigation of the radio source 3C 273 by the method of lunar occultations, Nature, 197, 1037-9, 1963.

[9] M. Schmidt, 3C 273: A star-like object with large red-shift, Nature 197, 1040, 1963.

[10] Quasi-Stellar Sources and Gravitational Collapse, Including the Proc. of the First Texas Symp. on Relativistic Astrophysics, eds Ivor Robinson et al (Chicago: University of Chicago Press), 1963.

[11] A.A. Michelson, Visibility of Interference-Fringes in the Focus of a Telescope, Publications of the Astronomical Society of the Pacific, 3, No. 16, 217-220, 1891.

[12] J.L. Pawsey, R. Payne-Scott, L. McCready, Radio-frequency energy from the sun, Nature 157, $158-9,1946$.

[13] L.L. McCready, J.L. Pawsey, R. Payne-Scott, Solar radiation at radio frequencies and its relation to sun spots, Proc Roy Soc A190, p357-375, 1947.

[14] W. Christiansen, J. Warburton, The distribution of radio brightness over the solar disk at a wavelength of 21 centimetres. III The quiet sun - two-dimensional observations, Aust. J. Phys. 8, 474-86, 1955.

[15] J.P. Wild J P, The Culgoora Radioheliograph - specification and general design, Proc. Institution of Radio \& Electrical Engineers Australia 28, 279-91, 1967.

[16] M. Ryle, D.D. Vonberg, Solar Radiation on 175 Mc/s, Nature 158, 339-340, 1946

[17] M. Ryle, A New Radio Interferometer and its Application to Observation of Weak radio Stars, Proc Roy Soc A211, 351-375, 1952.

[18] M. Ryle M, A. Hewish, The synthesis of large radio telescopes, MNRAS 120, 220, 1960.

[19] M. Ryle, A.C.Neville, A radio survey of the North Polar region with a 4.5 minute of arc pencilbeam system, MNRAS 125, p39, 1962.

[20] T. Pearcey, Use of Punched cards for Fourier Synthesis, CSIRO Division of Radiophysics, RPR121, 1953. 
[21] History of the Benelux Cross Project 1958 http://www.astron.nl/radioobservatory/public/history-wsrt/benelux-cross-antenna-project/benelux-cross-antenna-project

[22] J. P. Hamaker, J.D. O'Sullivan, J.E. Noordam, Image sharpness, Fourier optics, and redundantspacing interferometry, Optical Society of America, Journal, 67, 1122-1123, 1977

[23] P.J. Napier, A.R. Thompson, R.D. Ekers, The Very Large Array - design and performance of a modern synthesis radio telescope, in Proc. IEEE 71, 1295-1320, 1983.

[24] R.A. Perley et al, The Expanded Very Large Array: a new telescope for new science, The Astrophysical Journal Letters 739, L1, 2011.

[25] D. J. de Solla Price, Gears from the Greeks: the Antikythera Mechanism, a Calendar Computer from ca. 80 BC, Trans. Am. Phil. Soc., Vol. 64, part 7, 1974.

[26] D.J. de Solla Price, Little Science, Big Science, New York: Columbia University Press, 1963.

[27] M. Harwit, Cosmic Discovery - The Search, Scope \& Heritage of Astronomy, Basic Books Inc., New York, 1981.

[28] M.S. Livingston, J.P. Blewett,, Particle Accelerators, USA: McGraw Hill Book Co., 1962.

[29] K. Riesselmann, Deconstruction: Livingstone Plot, Symmetry 6, 30, 2009.

[30] G.E. Moore, Cramming more components onto integrated circuit, Electronics 38, no 8, 1965.

[31] E. Mollick, Establishing Moore's Law, IEEE Annals of the History of Computing 28, no 3, 2006.

[32] R.D. Ekers, Big and Small, in Int. Conf. Accelerating the Rate of Astronomical Discovery SPS5, IAU GA, August 11-14, 2009, Rio de Janeiro, Brazil, , 2009. 